\title{
A (heat) shock to the system promotes peripheral nerve regeneration
}

\author{
Ahmet Höke \\ Johns Hopkins School of Medicine, Baltimore, Maryland, USA.
}

\begin{abstract}
Peripheral nerves are easily damaged, resulting in loss of motor and sensory function. Recovery of motor and sensory function after peripheral nerve injury is suboptimal, even after appropriate surgical repair. This is due to the slow rate of axonal elongation during regeneration and atrophic changes that occur in denervated Schwann cells and target muscle with proximal lesions. One way to solve this problem is to accelerate the rate at which the axons regenerate. In this issue of the JCI, Ma and colleagues show that this can be achieved in mice by overexpression of heat shock protein 27, providing hope for enhanced functional recovery in patients after peripheral nerve damage.
\end{abstract}

Peripheral nerves are fragile and easily damaged, occurring, for example, with skin lacerations and bone fractures. Severe peripheral nerve injury requiring surgical intervention occurs in about $3 \%$ of all trauma cases admitted to emergency rooms (1). Recovery after peripheral nerve injury depends on a variety of critical factors. Experience with nerve injuries during World War II taught us that these factors include the type and location of the injury, the age of the patient, and the length of time that elapses before surgical repair is performed $(2,3)$. In terms of the type of injury, peripheral nerve injuries are classified as complete (wherein all the neurons traversing the site of injury are disrupted, causing total loss of distal motor or sensory function) or incomplete (wherein some of the neurons remain intact, meaning that some distal motor or sensory function is spared). Further, the injury may simply result in disruption or distortion of the myelin sheath, resulting in focal demyelination but leaving the axons intact, or cause damage to the axon itself. Over the years, most of the significant improvements in clinical outcome have been achieved by perfecting the surgical techniques used and performing nerve repairs early after the injury (4). With appropriate surgical intervention, clinical outcomes after distal nerve injuries are usually satisfactory, with both motor and protective sensory function ultimately being restored. However, with nerve lesions proximal to the CNS, functional

Conflict of interest: The author has declared that no conflict of interest exists.

Citation for this article: JClin Invest. 2011; 121(11):4231-4234. doi:10.1172/JCI59320. recovery in distal limb function is almost never achieved (5). The main limitation to functional recovery after proximal nerve injury is the relatively slow and fixed rate of axonal regeneration. On average, human peripheral nerves regenerate at a rate of approximately 1 inch per month. This rate is close to the slow axonal transport rate and is largely dictated by the need to move neurofilaments and microtubules, building blocks of axons, through the long axons (6, $7)$. As a consequence, appropriate repair of a distal ulnar nerve transection injury in the forearm can be expected to result in full recovery of hand function after 9 to 12 months, but injury to the same nerve in the brachial plexus in the axilla rarely ends in functional recovery, even after 2 to 3 years. In this issue of the JCI, Ma and colleagues identify a potential new target (heat shock protein 27 [Hsp27; also known as Hspb1]) to enhance functional recovery in patients after peripheral nerve damage, as they demonstrate that the rate at which axons regenerate is accelerated in transgenic mice overexpressing human Hsp27 (8). The authors claim that there is a critical period in mice after peripheral nerve damage (35 days), during which reinnervation must occur if functional recovery is to be achieved; overexpression of Hsp27 allowed reinnervation during this time.

\section{Problems in nerve regeneration}

The main question that arises from the observation that proximal nerve transection injury rarely results in functional recovery is does the axon loose its ability to regenerate if it does not reach its target in a timely manner, or do Schwann cells (the principle sup- porting glial cells in the peripheral nervous system) in the distal denervated nerve loose their ability to support regeneration if they are not innervated in a timely manner? This issue was settled by the seminal work of Fu and Gordon $(9,10)$, in which they modeled both chronic axotomy and chronic denervation in rats and asked what happens when a delayed nerve repair is carried out. Chronic axotomy refers to transection of the peripheral nerve and prevention of the axon from regenerating and innervating a target muscle; it can be performed to discern what happens to the ability of the neuron to regenerate if it does not make a timely connection to its target muscle. In contrast, chronic denervation refers to transection of the nerve and prevention of reinnervation of the distal nerve and target muscle with regenerating axons; the key question addressed by chronic denervation is what happens to the ability of Schwann cells to support regeneration. In the reports by Fu and Gordon $(9,10)$, after 6 months of chronic axotomy, in which transected tibial nerve axons were prevented from innervating a muscle, most of the axons retained their ability to regenerate and innervate a muscle when a delayed cross-repair was done into freshly transected peroneal nerve, indicating that neurons do not loose their innate ability to regenerate, even after prolonged periods of chronic denervation (9). However, in a reverse experiment, in which the distal peroneal nerve was left chronically denervated for 6 months, i.e., the peroneal nerve was transected just distal to trifurcation of the sciatic nerve and regeneration of proximal axons was prevented into the distal nerve segment, Schwann cells in the distal peroneal nerve lost their ability to support regeneration of freshly transected tibial axons (10), suggesting that if Schwann cells are not innervated in a timely manner, they no longer support regeneration and create a local environment in the distal peripheral nerve that is hostile to axonal regeneration (11). The changes that occur in chronically denervated distal nerves include Schwann cell atrophy, which results in loss of basal lamina and Schwann cell tubes $(12,13)$, and 


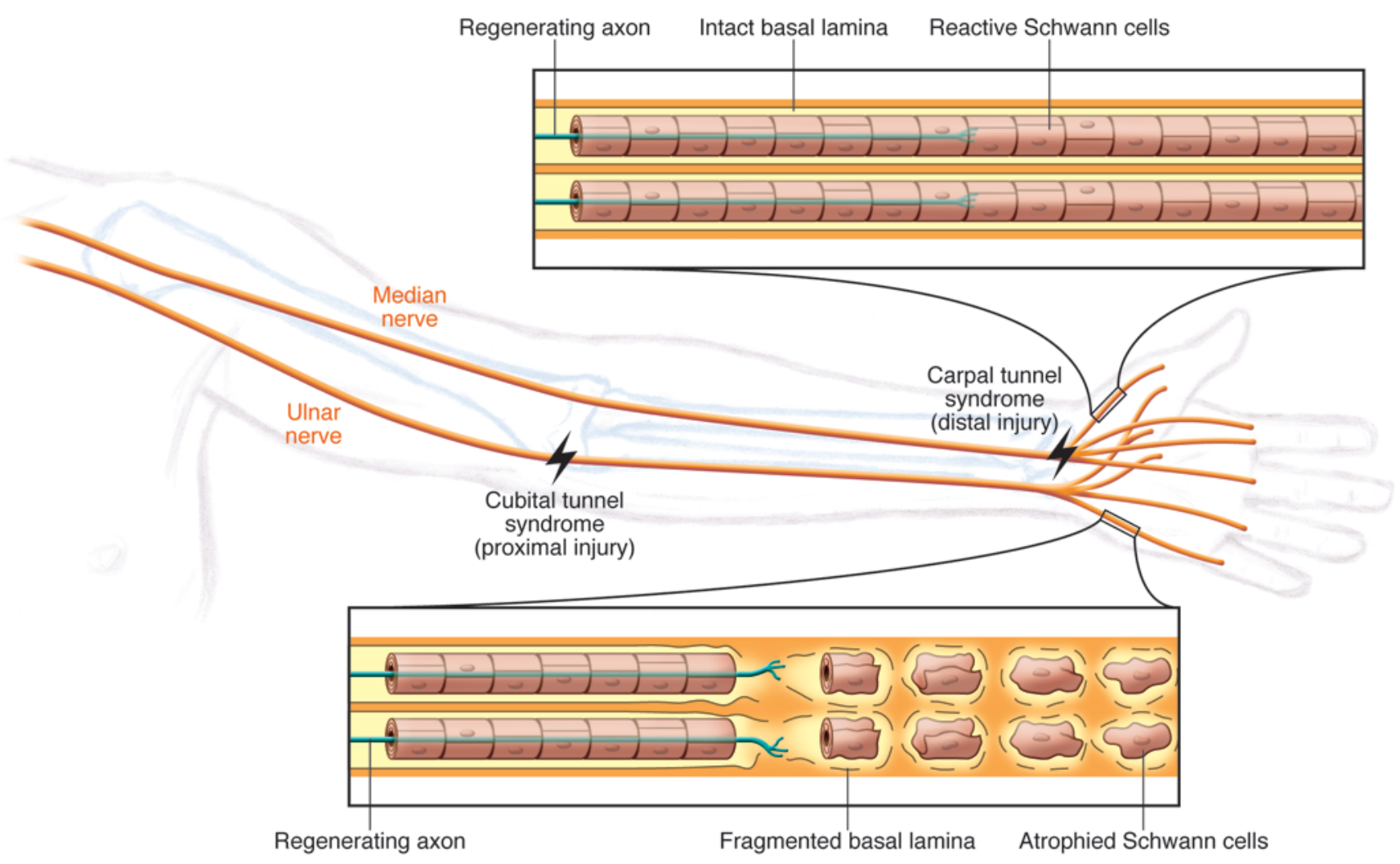

Figure 1

Challenges to successful regeneration with proximal injuries. Good sensory and motor recovery is likely if surgery is not delayed after a distal nerve injury, such as median nerve injury at the carpal tunnel. However, motor recovery after a more proximal injury, for example, to the ulnar nerve at the cubital tunnel in the elbow, is likely to be slow and incomplete due to the atrophic changes that take place in the distal portions of the nerve. One of the key reasons for this poor recovery of motor function is the slow rate of axonal regeneration; it takes too long for the injured axons to regenerate and connect with Schwann cells and muscle.

reduced expression of growth-promoting molecules, such as neurotrophic factors (14, 15). Coupled with an increase in expression of regeneration-inhibiting proteoglycans in the extracellular matrix $(16,17)$, the distal nerve environment in a chronically denervated nerve is akin to that of a "glial scar," which is one of the main impediments to regeneration in the CNS (Figure 1).

One of the limitations of the earlier models of regeneration after chronic denervation (10) was that they could not address the role of any changes that may occur in the denervated target muscle, although clinically it was suspected that if a muscle is not innervated in a timely manner (usually 2 years in humans), it loses its ability to be reinnervated. In a more recent study, Gordon and colleagues modeled chronic denervation in rat muscles and showed that, similar to chronic denervation in Schwann cells, muscle cells undergo irreversible changes if they are not reinnervated within 3 to 6 months, and functional recovery does not occur after that time (18).

\section{A "heat" shock solution to the problem}

So, how can we solve the problem of chronic denervation and lack of regeneration after proximal nerve lesions? There are two ways. One is to figure out how to keep the Schwann cells and muscle cells in a "reactive" state, so that Schwann cells can support regeneration as the axons slowly regenerate and muscle cells can accept reinnervation and formation of functional neuromuscular junctions. Another way to solve this problem would be to accelerate the rate at which axons regenerate, so that chronic denervation changes in the pathway (i.e., in Schwann cells) and target (i.e., the denervated muscle) do not occur. For this strategy to succeed with brachial plexus injuries, the rate of axonal regeneration needs to be increased to at least 2- to 3-fold faster than the natural rate of 1 inch per month.

We know that the rate of axonal regeneration can be accelerated, although previous attempts have resulted only in relatively modest increases. A classic example of an increase in the rate of regeneration has been achieved through conditioning lesion (19). That is, the rate of nerve regeneration is accelerated if a peripheral nerve is injured by crushing the nerve a week before a second more severe injury such as nerve transection. This effect depends on changes in gene expression in the dorsal root ganglia, and one of the primary transcription factors that is upregulated after peripheral axon injury is activating transcription factor 3 (ATF3) (20). Overexpression of ATF3 in dorsal root ganglion sensory neurons resulted in accelerated regeneration of sensory axons (21), but the effect was very modest, and the results await confirmation in a large animal model of long-distance regeneration after nerve repair.

In this issue of the JCI, Ma and colleagues used an unbiased bioinformatics approach to identify genes that play a key role in the regenerative response to nerve transection or crush injury in mouse dorsal root ganglion neurons and identified Hsp27 as a potential candidate to enhance the intrinsic 


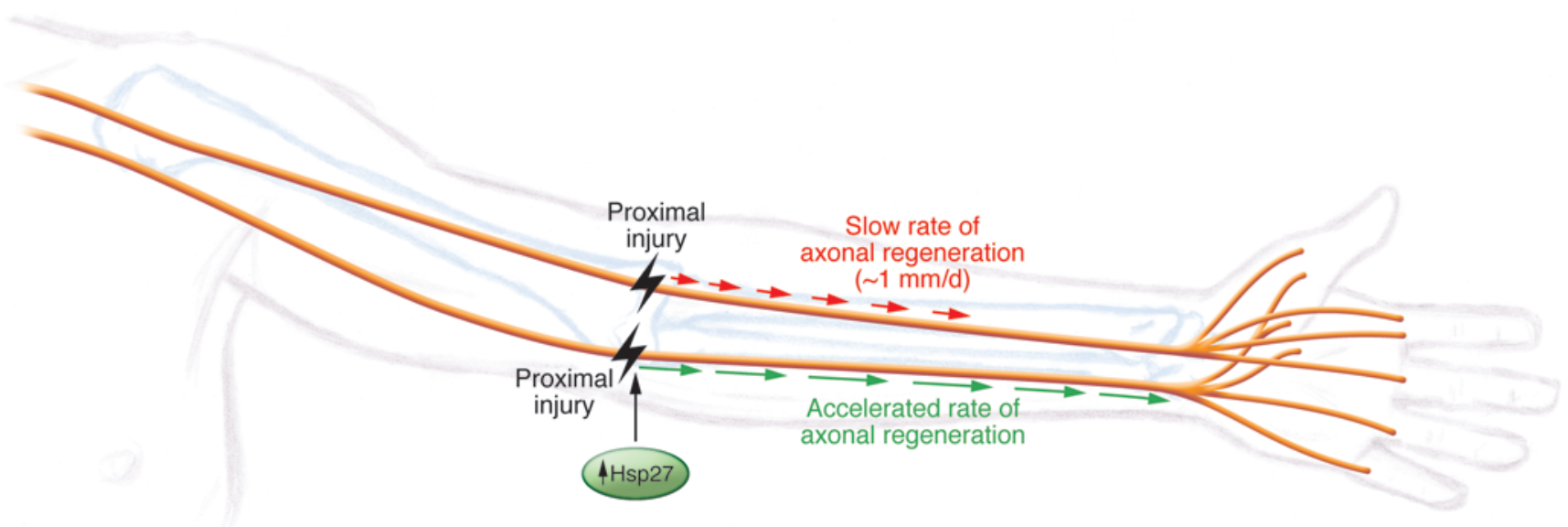

Figure 2

A "heat" shock to turbocharge the rate of regeneration. The work of Ma and colleagues (8) tries to solve the problem of poor recovery by accelerating the rate at which axons regenerate. Overexpression of human Hsp27 in sensory and motor neurons in mice resulted in an accelerated rate of axonal regeneration akin to "turbocharging" a snail's pace. This intervention is likely to be useful in any type of proximal nerve injury, such as severe cubital tunnel syndrome.

state of neurons for regeneration (8). Using transgenic mice that overexpressed human Hsp27, the authors demonstrated that the rate of axonal regeneration was enhanced in both motor and sensory axons at a rate similar to that observed with ATF3 overexpression (Figure 2). What is interesting in this study is that the authors found that wildtype littermates of the human Hsp27-overexpressing transgenic mice never regained full function of toe spreading after axotomy, even after 8 weeks, but that the transgenic mice exhibited proper reinnervation of the neuromuscular junctions and partial recovery of toe spread. The authors concluded that if the distal foot muscles are not reinnervated within 5 to 6 weeks, the neuromuscular junctions become difficult to reinnervate and thus there is a critical period in which reinnervation of the distal muscles has to take place. However, I believe that this conclusion is premature, as they used only one functional index, toe spread, instead of multiple outcome measures. As noted by others (reviewed in ref. 22), toe spread alone is not a reliable measure of full functional recovery after sciatic nerve transection and repair. Ma and colleagues also noted that 8 weeks after nerve transection and repair, neuromuscular junctions were not fully reinnervated in wild-type littermates but had fully recovered to control levels in the human Hsp27-overexpressing transgenic mice. The way the neuromuscular junctions were quantified needs verification, as only $80 \%$ of neuromuscular junctions were innervated in controls, which would be unusual and indicate a problem of neuromuscular innervation in this mouse background.

\section{Motor recovery in humans lags behind sensory recovery}

$\mathrm{Ma}$ and colleagues noticed that if repair was done soon after peripheral nerve damage in mice, recovery of both motor and sensory functions took place. However, if repair was delayed, although sensory function recovered, recovery of motor function was incomplete (8). A similar discrepancy in recovery of sensory versus motor function was observed in patients after surgical decompression of cubital tunnel syndrome (8). As noted by others in the field, Ma and colleagues demonstrated that recovery of sensory function occurred more readily compared with motor recovery after compressive nerve injury, such as occurs in carpal tunnel syndrome or cubital tunnel syndrome. Furthermore, Ma and colleagues provided a potential explanation for the poor motor recovery after cubital tunnel surgery. They demonstrated that, with repair after the onset of symptoms, motor recovery in individuals with cubital tunnel syndrome, in which the ulnar nerve is injured at the elbow, is not as good as that in individuals with carpal tunnel syndrome, in which the injury to the median nerve is more distal, at the wrist level. This observation is related to the importance of the distance that a nerve has to regenerate in order to reach its target. Ma and colleagues also confirmed earlier work (3) and showed that if repair is delayed, recovery of motor function is poor (8). Among the 20 patients with cubital tunnel syndrome, those who had surgery less than 10 months after the onset of symptoms had good recovery of motor function, while those who had surgery after that time had poor recovery. However, I believe that the data provided are preliminary, as details of the patient characteristics, including their full functional and electrophysiological evaluations, are not available. Out of the 20 patients with cubital tunnel syndrome that had surgery, 11 of the patients had very severe cubital tunnel syndromes, with complete denervation of the ulnar nerve-innervated intrinsic hand muscle, interosseus dorsalis. Since these patients were the most severely affected group, and, thus the least likely to recover, even after surgery, it is important to know whether they were in the group that had surgery in less than 10 months or in the group that was operated on 10 or more months after the onset of symptoms. Furthermore, it is unclear whether the functional evaluations of the patients were done in a blinded manner or not. Intuitively, it makes sense that the motor recovery after ulnar nerve decompression at the elbow may take longer and may not fully recover compared with median nerve decompression at the wrist. However, given the caveats, further work on this matter will be required to determine whether indeed this is the case.

\section{Clinical potential of key regulators of regenerative response}

The strength of the work of Ma and colleagues is their demonstration that an 
unbiased bioinformatics approach can yield useful therapeutic targets to enhance nerve regeneration (8). However, the current data on both ATF3 (21) and Hsp27 (8) show only modest improvements in the rate of axonal regeneration in short-term, short-distance nerve regeneration. Therefore, translation into a clinically useful target awaits confirmation in models of long-distance nerve repair in large animals. One thing that would be extremely interesting to investigate is whether overexpression of both ATF3 and Hsp27 in the same animal has an additive or synergistic effect in enhancing the rate of peripheral nerve regeneration. The group led by Clifford Woolf is at the forefront of this research and has the mice needed to examine this issue at hand. Combining their prior work with ATF3 (21) and current work on Hsp27 (8) should be a logical next step. Once a proof-of-concept demonstration with overexpression of both ATF3 and Hsp27 is shown to be a feasible solution, drug-screening assays for small molecules that may mimic their activities may lead to actual improvements in peripheral nerve regeneration.

\section{Acknowledgments}

Ahmet Höke's research is supported by NINDS, The Foundation for Peripheral Neuropathy, Merkin Family Foundation, and Adelson Medical Research Foundation.
Address correspondence to: Ahmet Höke, Johns Hopkins School of Medicine, 855 N. Wolfe Street, Rangos 248, Baltimore, Maryland 21205, USA. Phone: 410.955.2227; Fax: 410.502.5459; E-mail: ahoke@jhmi.edu.

1. Noble J, Munro CA, Prasad VS, Midha R. Analysis of upper and lower extremity peripheral nerve injuries in a population of patients with multiple injuries. J Trauma. 1998;45(1):116-122.

2. Sunderland S. Factors influencing the course of regeneration and quality of recovery after nerve suture. Brain. 1952;75(1):19-54.

3. Woodhall B, Beebe GW. Peripheral Nerve Regeneration: A Follow-up Study Of 3,656 World War II Injuries. Washington, DC, USA: US Government Printing Office; 1956.

4. Dvali L, Mackinnon S. The role of microsurgery in nerve repair and nerve grafting. Hand Clin. 2007; 23(1):73-81.

5. Brushart T. Nerve Repair. New York, New York, USA: Oxford University Press; 2011.

6. Grafstein B. Role of slow axonal transport in nerve regeneration. Acta Neuropathol. 1971; 5(suppl 5):144-152.

7. Hoffman PN, Lasek RJ. Axonal transport of the cytoskeleton in regenerating motor neurons: constancy and change. Brain Res. 1980;202(2):317-333.

8. Ma CHE, et al. Accelerating axonal growth promotes motor recovery after peripheral nerve injury in mice. J Clin Invest. 2011;121(11):4332-4347.

9. Fu SY, Gordon T. Contributing factors to poor functional recovery after delayed nerve repair: prolonged axotomy. J Neurosci. 1995;15(5 pt 2):3876-3885.

10. Fu SY, Gordon T. Contributing factors to poor functional recovery after delayed nerve repair: prolonged denervation. J Neurosci. 1995;15(5 pt 2):3886-3895.

11. Hoke A. Mechanisms of disease: what factors limit the success of peripheral nerve regeneration in humans? Nat Clin Pract Neurol. 2006;2(8):448-454.
12. Terenghi G, Calder JS, Birch R, Hall SM. A morphological study of Schwann cells and axonal regeneration in chronically transected human peripheral nerves. J Hand Surg Br. 1998;23(5):583-587.

13. Weinberg HJ, Spencer PS. The fate of Schwann cells isolated from axonal contact. J Neurocytol. 1978; 7(5):555-569.

14. Hoke A, Gordon T, Zochodne DW, Sulaiman OA. A decline in glial cell-line-derived neurotrophic factor expression is associated with impaired regeneration after long-term Schwann cell denervation. Exp Neurol. 2002;173(1):77-85.

15. Li H, Terenghi G, Hall SM. Effects of delayed reinnervation on the expression of c-erbB receptors by chronically denervated rat Schwann cells in vivo. Glia. 1997;20(4):333-347.

16. Heine W, Conant K, Griffin JW, Hoke A. Transplanted neural stem cells promote axonal regeneration through chronically denervated peripheral nerves. Exp Neurol. 2004;189(2):231-240.

17. Zuo J, Hernandez YJ, Muir D. Chondroitin sulfate proteoglycan with neurite-inhibiting activity is upregulated following peripheral nerve injury. $\mathrm{J} \mathrm{Neu}$ robiol. 1998;34(1):41-54.

18. Gordon T, Tyreman N, Raji MA. The basis for diminished functional recovery after delayed peripheral nerve repair. J Neurosci. 2011;31(14):5325-5334.

19. McQuarrie IG, Grafstein B, Gershon MD. Axonal regeneration in the rat sciatic nerve: effect of a conditioning lesion and of dbcAMP. Brain Res. 1977;132(3):443-453.

20. Tsujino $\mathrm{H}$, et al. Activating transcription factor 3 (ATF3) induction by axotomy in sensory and motoneurons: A novel neuronal marker of nerve injury. Mol Cell Neurosci. 2000;15(2):170-182.

21. Seijffers R, Mills CD, Woolf CJ. ATF3 increases the intrinsic growth state of DRG neurons to enhance peripheral nerve regeneration. J Neurosci. 2007;27(30):7911-7920.

22. Varejao AS, Melo-Pinto P, Meek MF, Filipe VM, Bulas-Cruz J. Methods for the experimental functional assessment of rat sciatic nerve regeneration. Neurol Res. 2004;26(2):186-194.

\title{
Do MDL-1+ cells play a broad role in acute inflammation?
}

\author{
Peter A. Ward \\ University of Michigan Medical School, Department of Pathology, Ann Arbor, Michigan, USA.
}

\begin{abstract}
Dengue virus (DV) reacts with myeloid DAP12-associating lectin-1 (MDL-1) on immature polymorphonuclear leukocytes. Interaction of DV with MDL-1 ${ }^{+}$ cells triggers systemic inflammatory response syndrome (SIRS) and dengue shock syndrome (DSS), with subsequent multi-organ failure. In this issue of the JCI, Cheung et al. find that sterile acute liver injury in mice is associated with the accumulation of MDL- $1^{+}$cells and that triggering of these cells by DV or an MDL-1-specific agonist antibody leads to SIRS, shock, and death. These findings may have broad mechanistic and therapeutic implications for the development of SIRS, sepsis, and shock in humans exposed to a wide array of infectious and non-infectious conditions.
\end{abstract}

Conflict of interest: The author has declared that no conflict of interest exists.

Citation for this article: J Clin Invest. 2011; 121(11):4234-4237. doi:10.1172/JCI60122.
Localized activation of the innate immune system is critical for host defenses against invasive pathogens and for repairing tissues damaged by trauma, ischemia/reperfusion injury, or chemical-induced injury (1). In some instances, the localized response to infection or sterile tissue damage becomes progressive, escalating to systemic inflammatory response syndrome (SIRS), a condition that can progress to shock, multi-organ failure, and death (2). The production of proinflammatory cytokines and ROS by activated myeloid lineage cells, especially polymorphonuclear leukocytes (PMNs) and macrophages, is a key component of SIRS. However, the cascade of events that causes SIRS to progress to shock, defined clinically as systemic hypotension, is not well defined. Myeloid lineage cell produc- 